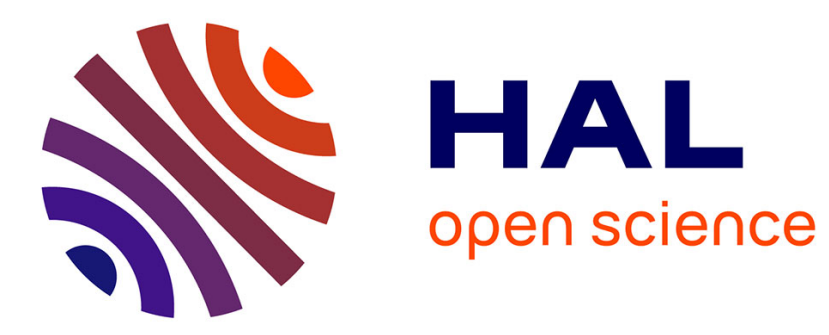

\title{
One-way rotation of a molecule-rotor driven by a shot noise
}

\author{
Jorge Echeverria, Serge Monturet, Christian Joachim
}

\section{To cite this version:}

Jorge Echeverria, Serge Monturet, Christian Joachim. One-way rotation of a molecule-rotor driven by a shot noise. Nanoscale, 2014, 6 (5), pp.2793-2799. 10.1039/c3nr05814j . hal-01712782

\section{HAL Id: hal-01712782 \\ https://hal.science/hal-01712782}

Submitted on 19 Jun 2018

HAL is a multi-disciplinary open access archive for the deposit and dissemination of scientific research documents, whether they are published or not. The documents may come from teaching and research institutions in France or abroad, or from public or private research centers.
L'archive ouverte pluridisciplinaire HAL, est destinée au dépôt et à la diffusion de documents scientifiques de niveau recherche, publiés ou non, émanant des établissements d'enseignement et de recherche français ou étrangers, des laboratoires publics ou privés. 


\title{
One-way rotation of a molecule-rotor
}

\section{driven by a shot noise}

\author{
Jorge Echeverria*, Serge Monturet and Christian Joachim \\ Nanosciences Group \& MANA Satellite, CEMES/CNRS, 29 rue Jeanne Marvig, 31055 \\ Toulouse, France.
}

KEYWORDS: Single molecule motor, rotation mechanism, shot noise, excited states.

\begin{abstract}
:
The shot noise of the tunneling current passing through a molecule-motor can sustain its rotor one-way rotation when virtually populating its excited states by tunneling inelastic excitations. No need for a ratchet like ground state rotation potential energy of the rotor. Just a relative shift in energy between the maxima of this ground state and the minima of the excited states used is required. The rotor speed of rotation and its rotation direction are both controlled by this shift pointing out the need of a careful design of both the ground and excited states of the next generation of molecule-motor to be able to generate a motive power.
\end{abstract}




\section{INTRODUCTION}

Since the first observation of the rotation of a single molecule adsorbed on a surface, ${ }^{1}$ studies on single molecule mechanical machineries on a surface have expanded in many directions such as molecule-gears, ${ }^{2,3}$ molecule-motors ${ }^{4,5}$ and molecule nano-vehicles. ${ }^{6,7}$ Nowadays, there are several possibilities to drive the rotation of a single molecule rotor when adsorbed on a metallic surface, like pushing on the rotor by an STM or AFM tip, ${ }^{2,8}$ feeding the rotor using inelastic electron tunnelling effects via an STM tip ${ }^{9,10}$, applying an oscillating electric field to the rotor to synchronise its rotation with the large oscillation period of this field, ${ }^{11,12,13}$ or powering the rotor with light. ${ }^{4}$

Aside from a tip pushing, i.e. when the operator decides the rotation direction of rotor, ${ }^{2}$ in the others cases mentioned above the conditions for a one way rotation of a single molecule-rotor in a molecule-motor have not yet been clarified. Currently, the literature is fairly following two directions: (a) the introduction of an intramolecular ratchet effect which is usually characterized by plotting the potential energy variation of the rotor as a function of its rotation angle ${ }^{14}$ and (b) the control of the interactions between the rotor and a specific external driving force to break micro-reversibility. ${ }^{11}$ In this article, following the recent demonstration of a control rotation of a single molecule motor driven by a tunnelling current, ${ }^{14}$ we demonstrate by constructing a general model from this example that the conditions for a one-way rotation of a single molecule motor are between (a) and (b). From (a), there is a need to break the symmetry of the rotational potential energy surface of the rotor as a function of its rotation angle and from (b) there is a need for the rectification of some random excitation process of the rotor to drive the rotation. At the origin of this study, our molecule motor is a piano-stool organometallic complex composed of a five-arm rotor mounted on a tripod stator as presented in Figure 
$1^{14,15,16}$ This molecular motor was recently operated by STM on a $\mathrm{Au}(111)$ surface yielding a step-by-step one-way rotation, both clockwise and counter-clockwise, under a tunnelling current. The direction of rotation was selected by positioning the STM tip either on a truncated or a non-truncated arm of the rotor.

In this paper, we first build up a simple model for the Figure 1 motor to analyse the general requirements for the rotor of a single molecule-motor to rotate one-way in a controllable manner. Then, a complete numerical analysis of the rotation dynamics of the corresponding rotor is performed, paying special attention to the average driving force and to the role played by the electronic excited states of the molecule. Finally, we demonstrate how to drive a one-way rotation using the shot noise of the tunnelling current passing through the molecule-motor explaining also how to control the way of rotation.

\section{DISCUSSION}

A simple mechanical model for a molecule-motor. The electronic ground and first excited states potential energy surfaces of the Fig. 1 motor mainly depend on 9 angles: the top rotor rotation angle $\theta$ around the central Ru axis, the 5 flipping angles $\varphi_{1}$ to $\varphi_{5}$ of the 5 rotor phenyl arms and the 3 stator legs deformation angles $\chi_{1}$ to $\chi_{3}$. Despite many others degrees of freedom contribute to the mechanics of this motor, their role is minor as compared to those 9 principal ones. Even with only those 9 coordinates, the corresponding $\mathrm{E}_{0}\left(\theta, \varphi_{1}, \ldots \varphi_{5}, \chi_{1}, . ., \chi_{3}\right)$ ground state potential energy surface is considerably difficult to master. Starting from one of the possible $E_{0}\left(\theta, \varphi_{1}, \ldots \varphi_{5}, \chi_{1}\right.$, .., $\left.\chi_{3}\right)$ energy minima, a simple way to model the rotation mechanisms is to step by step increase $\theta$ while minimizing the molecule motor potential energy for each $\theta$. This 
delivers a $\theta$ parametric rotation trajectory on the $\mathrm{E}_{\mathrm{o}}\left(\theta, \varphi_{1}, \ldots \varphi_{5}, \chi_{1}, . ., \chi_{3}\right)$ manifold. For the Fig. 1 motor, this trajectory was calculated using the ASED+ semi-empirical technique. Along this rotation trajectory, the resulting $\mathrm{E}_{\mathrm{o}}(\theta)$ potential energy curve is highly asymmetric, with a potential barriers height of $\Delta \mathrm{V}=0.25 \mathrm{eV}$ as presented in Fig. 2. On $\mathrm{E}_{0}$, the combination of the rotor upper $C_{5}$ and the stator lower $C_{3}$ concurrent symmetries ${ }^{17}$ leads to 15 potential wells per turn. This periodic series of barriers is due to the interaction between the 5 phenyl rings of the rotor arms and the 3 motor legs bound to the surface. During the rotation and every time one arm interacts with one leg, the corresponding phenyl ring flips in order to avoid the leg and this leg is consequently deformed. On the Fig. 2 curve, this results in a ratchet-like potential energy variation. Calculations with a 4 arms rotor are qualitative identical (see Fig. S1 in Supporting Information) MAY BE NOT NECESSARY and Also THE CORRESPONDING Supporting Information. However, this asymmetry, constructed along the rotation trajectory, is artificial because it is created by forcing the rotation in a given direction. There is no asymmetry when passing from one minimum to the next on $\mathrm{E}_{\mathrm{o}}\left(\theta, \varphi_{1}, \ldots \varphi_{5}\right.$, $\left.\chi_{1}, . ., \chi_{3}\right)$ and, thus, no breaking of the micro-reversibility principle. Therefore, by forcing a $\theta$ rotation, we are anticipating the good functioning of the molecule motor but not demonstrating it. In fact, with the initial objective of determining the conditions for a one-way rotation, an ideal rotation trajectory was created on $\mathrm{E}_{0}\left(\theta, \varphi_{1}, \ldots \varphi_{5}, \chi_{1}, . ., \chi_{3}\right)$ as if the rotor was already rotating one way instead of finding the optimized excitation process to approach this ideal rotation trajectory.

To illustrate how a saw-tooth like rotation trajectory can come out from an $a$ priori symmetric potential energy surface, let us consider the rotation of a single phenyl of the 2,2'-dibromobiphenyl molecule around its central $\mathrm{C}-\mathrm{C}$ bond (Figure $3 \mathrm{a}$ ). The $\theta$ 
rotation angle is here defined by the dihedral $\mathrm{C} 1, \mathrm{C} 2, \mathrm{C} 3, \mathrm{C} 4$ angle and the $\varphi$ torsion angle by the $\mathrm{C} 2, \mathrm{C} 3, \mathrm{C} 4, \mathrm{Br} 5$ dihedral angle (Figure $3 \mathrm{a}$ ). To build up the $\mathrm{E}_{\mathrm{o}}(\theta, \varphi)$ potential energy surface presented in Fig. 3b, the molecule potential energy was calculated as a function of $(\theta, \varphi)$ without optimization of the molecular geometry by means of the AM1 semi-empirical technique. This surface is periodic in $(\theta, \varphi)$ and provides a map of one of those periods including also two energy minima A and B. There is a central energy maximum corresponding to the $\mathrm{Br}-\mathrm{Br}$ repulsion. This maximum is symmetric, as expected from the original symmetry of the molecule.

With no external forces applied, this molecule remains in one of the $\mathrm{E}_{0}(\theta, \varphi)$ energy minima. When a $\theta$ rotation imposes to rotate from $\mathrm{A}$ to $\mathrm{B}$ and the molecule energy is minimized at each $\theta$ steps of $1^{\circ}$, the Fig. $3 \mathrm{~b}$ top surface trajectory is obtained. If now, starting from $\mathrm{B}$, the molecule is forced to return back to A, the Fig. $3 \mathrm{~b}$ down trajectory is obtained. Following both trajectories by plotting the corresponding potential energy variations as a function of $\theta$, the Fig. $3 \mathrm{c}$ saw-tooth like curves are obtained. Of course, a unique minimum energy path is expected after a complete search with a full optimization of the reaction coordinate of a non-forced rotation. Notice that an asymmetric energy rotation trajectory with two different pathways between A and B is also obtained when the partial optimization involves all the molecular degrees of freedom but the rotation angle. However, if this torsion angle is the only degree of freedom allowed during the optimization, the rotation energy profile is then symmetric.

This example illustrates the extreme care required in selecting the good degree of freedom to describe the mechanics of a single molecule motor. Since the number of degrees of freedom is generally quite large, the ones to consider, the ones to minimize and the ones to freeze must be very well chosen to model in a full generality the 
intramolecular physics involved. For the Fig. 1 like molecule-motor (and for many others of the same kind), a minimum of two degrees of freedom, here the $\theta$ rotation angle and a generic torsion angle $\varphi$, must be considered and not only $\theta$ as used in Fig. 2 . For the Fig. 1 motor, this torsion angle $\varphi$ can be defined by the dihedral angle between a given phenyl ring of the rotor and the central cyclopentadienyl ring. In this case, a model $\mathrm{E}_{\mathrm{o}}(\theta, \varphi)$ ground state can be written:

$$
E_{0}(\theta, \varphi)=V_{0} \sin ^{2}(\theta+\varphi) \cos ^{2}(\theta+\varphi) e^{-\varphi^{2}}+k \varphi^{6}
$$

Instead of the 15 minima of the Fig. 1 molecule motor, we have here limited the number of minima to 4 per turn to simplify the analysis. It can be easily extended to 15 or more for molecule motor with a larger number of arms. During the rotor rotation, the steric repulsion between a rotor phenyl and one of the three stator legs is taken into account in (1) by a $\varphi^{6}$ term (with $k=5 \cdot 10^{-3}$ ). $V_{0}$ models the energy barrier height and $k$ the strength of the deformation. This $\mathrm{E}_{\mathrm{o}}(\theta, \varphi)$ periodic potential energy surface is presented in Figure 4. When a $\theta$ rotation is forced on this surface, the energy variation along the rotation trajectory minimized by using the Nudged Elastic Band (NEB) method ${ }^{18}$ is similar to the one presented in Figure 1b (see Figure S2 in Supporting Information).

The $\boldsymbol{E}_{\boldsymbol{n}}$ electronic excited states involved in the rotation are certainly composed of several unoccupied molecular orbitals and the corresponding $\boldsymbol{E}_{n}(\theta, \varphi)$ potential energy surfaces associated to the rotation are rather complex. ${ }^{14}$ Since our goal here is to understand how the excited states can be involved to reach an unidirectional rotation, we can select (1) for the analytic expression of the first excited state $\boldsymbol{E}_{\boldsymbol{l}}(\theta, \varphi)$ and include a shifting term in this $\boldsymbol{E}_{\boldsymbol{l}}(\boldsymbol{\theta}, \boldsymbol{\varphi})$ to change the angular position of its minima relative to $E_{0}(\theta, \varphi)$ 
The molecule-motor equation of motion. After the construction of a general model for the potential energy surfaces of a molecule motor, the next step is to construct its general system of motion equations. A preparation of the molecule motor in a coherent non-stationary quantum superposition of states by mixing its ground and some molecule electronic excited states must be enough to provide the energy required to drive the motion. ${ }^{19}$ This preparation has to be cyclic in time since the rotor will lose rapidly the coherence of this initial preparation due to the large number of internal degrees of freedom of the molecule-motor and to the finite lifetime of the excited states. For the two angles used in our molecular motor model, the system of semi-classical equations of motion can be written:

$$
\begin{aligned}
& I \frac{d^{2}\langle\theta\rangle}{d t^{2}}=-\nabla_{\theta}\left(\sum \mid c_{n}(t)^{2} E_{n}(\theta, \varphi)\right)-\gamma a^{2} \frac{d\langle\theta\rangle}{d t}+F(t) a \\
& I \frac{d^{2}\langle\varphi\rangle}{d t^{2}}=-\nabla_{\varphi}\left(\sum \mid c_{n}(t)^{2} E_{n}(\theta, \varphi)\right)-\gamma a^{2} \frac{d\langle\varphi\rangle}{d t}+F(t) a
\end{aligned}
$$

where the molecular rotor is described by a disk of mass $\mathrm{m}=600 \mathrm{amu}$ and of radius $\mathrm{a}=$ $1 \mathrm{~nm}$, with an extremely small $I=10^{-42} \mathrm{~kg} \mathrm{~m}^{2}$ momentum of inertia with respect to $\theta$ and $\gamma$ being the friction coefficient of the rotor motion. Notice that in (3), the momentum of inertia of the rotation along $\varphi$ is supposed to be smaller (around $10^{-44} \mathrm{~kg}$ $\mathrm{m}^{2}$ ) than in (2). However, we have observed that this difference has very little effect on the dynamic behavior of the system. Accordingly, we have used the same value of $I=$ $10^{-42} \mathrm{~kg} \mathrm{~m}^{2}$ in both (2) and (3).

The coherent non-stationary preparation is described in (2) and (3) by the $c_{n}(t)$ summation over the $\boldsymbol{E}_{\boldsymbol{n}}(\boldsymbol{\theta}, \boldsymbol{\varphi})$ ground and electronic excited states potential energy surfaces. If the superposition coherence is step by step preserved along the rotation 
time, a semi-classical motion will result based on the multiple potential energy surfaces $\boldsymbol{E}_{\boldsymbol{n}}(\boldsymbol{\theta}, \boldsymbol{\varphi})$ employed for the dynamics and according to the $\boldsymbol{c}_{\boldsymbol{n}}(\boldsymbol{t})$ amplitudes of the superposition. But such a coherence is very difficult to preserve on the long run even by a cyclic re-preparation of the superposition. All the $c_{n}(t)$ for $n>0$ will progressively decay in time towards the ground state with an average decay rate $\boldsymbol{\beta}$. In (2) and (3), the external driving force $\boldsymbol{F}(\boldsymbol{t})$ is there to compensate for this decoherence and relaxation to sustain a rotation.

Considering the $\boldsymbol{E}_{0}(\theta, \varphi)$ ground state, the $\boldsymbol{E}_{1}(\theta, \varphi)$ excited state and the decay rate $\beta$, a new system of equations can be derived from (2) and (3) to describe molecule motor dynamics taking into account the total decoherence of the superposition included in (2) and (3):

$$
\begin{aligned}
& I \frac{d^{2} \theta}{d t^{2}}=\sum_{i}-\left(1-e^{-\beta}\right) \Gamma\left(t-t_{i}\right) \nabla_{\theta} E_{0}(\theta, \varphi) \sum_{i}-e^{-\beta} \Gamma\left(t-t_{i}\right) \nabla_{\theta} E_{1}(\theta, \varphi)-\eta a^{2} \frac{d \theta}{d t}+\sum_{i} F a \Gamma\left(t-t_{i}\right) \\
& I \frac{d^{2} \varphi}{d t^{2}}=\sum_{i}-\left(1-e^{-\beta t}\right) \Gamma\left(t-t_{i}\right) \nabla_{\varphi} E_{0}(\theta, \varphi) \sum_{i}-e^{-\beta} \Gamma\left(t-t_{i}\right) \nabla_{\varphi} E_{1}(\theta, \varphi)-\eta a^{2} \frac{d \varphi}{d t}+\sum_{i} F d \Gamma\left(t-t_{i}\right)
\end{aligned}
$$

where $\Gamma\left(t-t_{i}\right)$ is a time shifted step function that permits the force $F$ to act at a given time series $t_{i}$ and to reset the excited state preparation with a ground state total depopulation. Therefore, there is in (4) and (5) no more coherence between the ground and the excited states as compared to (2) and (3).

Before going to the full optimisation of $F(t)$ to drive the rotor one way, let us comment on what happens when this rotor is driven only in its ground state by an external random force $F(t)$. In this case, the system (4) and (5) simply reduces to the system: 


$$
\begin{aligned}
& I \frac{d^{2} \theta}{d t^{2}}=-\nabla_{\theta} E_{0}(\theta, \varphi)-\gamma a^{2} \frac{d \theta}{d t}+F(t) a \\
& I \frac{d^{2} \varphi}{d t^{2}}=-\nabla_{\varphi} E_{0}(\theta, \varphi)-\gamma a^{2} \frac{d \varphi}{d t}+F(t) a
\end{aligned}
$$

With this system, we have tested whether a one-way rotation trajectory can be stabilized on $\boldsymbol{E}_{\boldsymbol{0}}(\boldsymbol{\theta}, \boldsymbol{\varphi})$ using a simple Gaussian distributed $F(t)$ driving force. This force was applied in average, half time on (6) and half time on (7). The system (6) and (7) was solved using a Verlet-like algorithm based on the finite differences method. Due to the extremely small $I$ value, much care have been taken to ensure the stability of the numerical solutions. For a given $V o$ value and exploring different friction parameters relative to the average strength of $F(t)$, it was not possible to construct a $\theta$ trajectory on $\boldsymbol{E}_{0}(\theta, \varphi)$ corresponding to a one-way rotation of the rotor even if $F(t)$ is time correlated. This fact is in good agreement with the general behavior of Brownian motors, which cannot undergo directed motion in the presence of only one source of anisotropy. ${ }^{20}$

Driving a one-way rotation with a shot noise. In a molecular tunneling junction, the tunneling current intensity is the result of billions of electron transfers per second happening at random time. The temperature independent statistical time distribution of this random process is a Poisson distribution. ${ }^{21}$ Its first momentum gives the tunneling current intensity through the molecule depending on the bias voltage used whereas its second momentum measures the time fluctuations of this intensity and is characteristic of a shot noise. The force $F$ during each excitation in (4) and (5) can be constant for all random distributed $t_{i}$ or be also random in strength following for example a Gaussian distribution. The first case is equivalent to consider that each electron transfer event through the molecule is inelastically active. However, this is certainly not the case in a molecular tunnel junction. A Gaussian distribution for $F$ 
along the random $t_{i}$ represents better the low efficiency of an inelastic tunneling excitation. By selecting a Poisson time-dependent excitation distribution at $\mathrm{t}_{\mathrm{i}}$, the energy is provided to the molecule-motor by the shot noise. Furthermore, each time $t_{i}$ a zero mean Gaussian distributed $F$ is applied to the molecule, it must act on $\theta$ or $\varphi$ with an equal probability over time.

Unlike (6) and (7) using only the ground state of a molecule-motor, a one-way rotation trajectory is now obtained with (4) and (5) using the above described Poisson noise and with both $E_{0}(\theta, \varphi)$ and $E_{1}(\theta, \varphi)$ fully symmetric in $\theta$ and $\varphi$. As presented in Fig. 5, the necessary condition for such a rotation is a shift of the $\boldsymbol{E}_{1}(\theta, \varphi)$ minima relative to the $\boldsymbol{E}_{0}(\theta, \varphi)$ ones. The rotation stops when there is a coincidence between the $\boldsymbol{E}_{l}(\theta, \varphi)$ minimum and $\boldsymbol{E}_{0}(\theta, \varphi)$ maximum or between the $\boldsymbol{E}_{1}(\theta, \varphi)$ and $\boldsymbol{E}_{\boldsymbol{0}}(\theta, \varphi)$ minima. Notice that such behavior was previously reported in another context for the directed transport of Brownian particles in a double symmetric potential ${ }^{22}$.

According to Fig. 5, a change in the rotation direction is obtained by changing the min-max relative shift between $E_{0}(\theta, \varphi)$ and $\boldsymbol{E}_{1}(\theta, \varphi)$. This was observed experimentally with the Fig. 1a molecule-motor by locating the tip apex of the STM on different rotor arms to be coupled to different excited states of the molecule at the same positive bias voltage range. ${ }^{14}$ This gives a design rule for new molecule-motors with a possible reversible choice of the rotation direction since excited states with different min-max relative shifts with respect to the ground state can be reached depending on the location of the excitation. Knowing the detailed topology of the excited electronic states potential energy surface of a molecule-motor becomes now an important part of its 
design. For instance, an elegant example of a molecule able to shift its min-max using hydrogen bonds was recently reported by L. M. Frutos, D. Sampedro et al. ${ }^{23}$

With the same barrier height $(0.25 \mathrm{eV})$ in $\boldsymbol{E}_{\boldsymbol{o}}(\theta, \varphi)$ and $\boldsymbol{E}_{\mathbf{1}}(\theta, \varphi)$, a moleculemotor of the type presented Fig. 1 is able to rotate one way for an average Gaussian $F$ between 0.02 and $0.2 \mathrm{pN}$. The necessary condition is that the $\boldsymbol{E}_{\boldsymbol{0}}(\theta, \varphi)$ and $\boldsymbol{E}_{\mathbf{1}}(\theta, \varphi)$ minmax must be shifted as discussed above and presented in Fig.5. The average time interval between two $t_{i}$ that forges the Poisson distribution in (4) and (5) must also be tuned in order to get a stable unidirectional rotation. For example, according to the solution of (4) and (5) and for an excited state decay time $\beta^{-1}$ of 1 ns, the rotor of the molecule-motor rotates one-way for an average time separation between two $t_{i}$ larger than $0.35 \mathrm{~ns}$, reaching a maximum rotation speed at a $1.25 \mathrm{~ns} \mathrm{t}_{\mathrm{i}}$ interval. After this threshold, the average speed decays exponentially due to the decrease in the energy supplied to the molecule per unit of time as presented in Fig. 6a. Therefore, when $\boldsymbol{\beta}$ decreases, that is for very long $\boldsymbol{E}_{1}(\theta, \varphi)$ life times, it is necessary to increase the average excitation time further in order to keep control of the rotation. The control of the rotation is better when the average time interval between two $t_{i}$ is long enough as compared to the lifetime of the excited state (here longer than $2.5 \mathrm{~ns}$ ). For example, an average interval of $0.35 \mathrm{~ns}$ and of $7 \mathrm{~ns}$ between 2 consecutive $t_{\mathrm{i}}$ leads to similar rotation velocities $\left(\sim 7.5 \cdot 10^{6}\right.$ turns/s). This corresponds to a tunneling current intensity below 1 nA for this life time. Notice that the energy provided to the molecule-motor in the first case is twenty times larger than in the second (VERY GOOD, HOW TO EVALUATE THIS ENERGY? SEE THE NEXT RED REMARK BELOW). A representation of the two cases is presented in Fig. 6 b. 
Our model assumes that the relaxation process occurs between $\boldsymbol{E}_{\mathbf{1}}(\boldsymbol{\theta}, \boldsymbol{\varphi})$ and $\boldsymbol{E} \boldsymbol{o}(\theta, \varphi)$ whatever their energy difference. Therefore, it is not the absolute energy difference between $\boldsymbol{E} o(\theta, \varphi)$ and $\boldsymbol{E}_{1}(\theta, \varphi)$ in (4) and (5) which provide the energy for a one way rotation (AND SO What it is ..?). Furthermore, the barrier height along their respective potential energy surface of the states controls the rotor average velocity. We have employed for the simulations the value previously calculated with the ASED+ semi-empirical method, which is $0.25 \mathrm{eV}$ for the ground state, for a calculated average velocity of $3.43 \cdot 10^{7}$ turns/s. Increasing the energy barrier of both states up to $0.44 \mathrm{eV}$ leads to a slightly slower rotation $\left(3.28 \cdot 10^{7}\right.$ turns/s). However, when decreasing the barrier in $\boldsymbol{E}_{\boldsymbol{o}}(\theta, \varphi)$ to $0.25 \mathrm{eV}$ while keeping $\boldsymbol{E}_{\mathbf{1}}(\theta, \varphi)$ at $0.44 \mathrm{eV}$, a smaller average velocity is calculated $\left(2.25 \cdot 10^{7}\right.$ turns/s). In contrast, a barrier of $0.44 \mathrm{eV}$ in $\boldsymbol{E}_{\boldsymbol{0}}(\boldsymbol{\theta}, \boldsymbol{\varphi})$ and of $0.25 \mathrm{eV}$ in $\boldsymbol{E}_{1}(\theta, \varphi)$ leads to a large average velocity of $4.11 \cdot 10^{7}$ turns/s. Further modifications of the energy barriers must be accompanied by a new optimization of the average time interval between $2 t_{i}$ and of the average Gaussian force in order to keep the one-way rotation.

\section{CONCLUSIONS}

Formally equivalent to a particle in solution, which is subject to a thermal noise due to random collisions with solvent molecules, ${ }^{20}$ a surface-mounted molecule in ultra high vacuum can be exposed to a shot noise coming from the STM tip. This noise has been recently used to drive the motion of a single molecule motor. ${ }^{14}$ Inspired by this recent achievement, we have carried out a systematic study of the dynamics of a single molecule motor model driven by a shot noise to model a tunneling current inelastic excitation. The use of an excited state in driving the rotation of the molecule motor 
allows the conversion of a Poisson time-distributed applied force to a controlled unidirectional motion. For a barrier height of $0.25 \mathrm{eV}$, the motor reaches a maximum speed of $4.11 \cdot 10^{7}$ turns/s. Furthermore, the direction of rotation of a molecule-motor can be reversed depending on the characteristics of the excited state involved in the process. We have demonstrated that a molecule without an intrinsic asymmetry can reconstruct a one-way rotation by using its internal characteristics. These findings will help the optimization of the chemical structure of new molecule-motors, not only to rotate one-way but certainly also to develop a true motive power to be able to move atoms, small molecules or to drive cooperatively a train of solid state nano-gears.

\section{METHODS}

The rotation potential energy profile of the molecule motor ground state was obtained by means of the ASED+ semi-empirical technique. ${ }^{24}$ Rotation energy profile of 2,2'-dibromobiphenyl, partial geometry optimizations and scan calculations on this molecule to build the potential energy surfaces were done with the semi-empirical AM1 method $^{25}$ as implemented in Gaussian 09. ${ }^{26}$ Numerical simulations were carried out with a home-built program that solves the dynamics equations by means of a Verlet-like algorithm based on the finite differences method. 


\section{REFERENCES}

1. Gimzewski, J.; Joachim, C.; Schlittler, R.; Langlais, V.; Tang, H.; Johannsen, I. Rotation of a single molecule within a supramolecular bearing. Science 1998, 281, 531533.

2. Manzano, C.; Soe, W.-H.; Wong, H. S.; Ample, F.; Gourdon, A.;

Chandrasekhar, N.; Joachim, C. Step-by-step Rotation of a Molecule-gear Mounted on an Atomic-scale Axis. Nature Materials 2009, 8, 576-579.

3. Frantz, D. K.; Linden, A.; Baldridge, K. K.; Siegel, J. S. Molecular Spur Gears Comprising Triptycene Rotators and Bibenzimidazole-Based Stators. J. Am. Chem. Soc. 2012, 134, 1528-1535.

4. Delden, R. A. van; Wiel, M. K. J. ter; Pollard, M. M.; Vicario, J.; Koumura, N.; Feringa, B. L. Unidirectional Molecular Motor on a Gold Surface. Nature 2005, 437, 1337-1340.

5. Zheng, X.; Mulcahy, M. E.; Horinek, D.; Galeotti, F.; Magnera, T. F.; Michl, J. Dipolar and Nonpolar Altitudinal Molecular Rotors Mounted on an Au(111) Surface. $J$. Am. Chem. Soc. 2004, 126, 4540-4542.

6. Shirai, Y.; Morin, J.-F.; Sasaki, T.; Guerrero, J. M.; Tour, J. M. Recent progress on nanovehicles. Chem. Soc. Rev. 2006, 35, 1043-1055.

7. Joachim, C.; Rapenne, G. Molecule Concept Nanocars: Chassis, Wheels, and Motors? ACS Nano 2013, 7, 11-14.

8. Pawlak, R.; Fremy, S.; Kawai, S.; Glatzel, T.; Fang, H.; Fendt, L.-A.; Diederich, F.; Meyer, E. Directed Rotations of Single Porphyrin Molecules Controlled by

Localized Force Spectroscopy. ACS Nano 2012, 6, 6318-6324.

9. Nickel, A.; Ohmann, R.; Meyer, J.; Grisolia, M.; Joachim, C.; Moresco, F.; Cuniberti, G. Moving Nanostructures: Pulse-Induced Positioning of Supramolecular Assemblies. ACS Nano 2013, 7, 191-197.

10. Kudernac, T.; Ruangsupapichat, N.; Parschau, M.; Maciá, B.; Katsonis, N.; Harutyunyan, S. R.; Ernst, K.-H.; Feringa, B. L. Electrically Driven Directional Motion of a Four-wheeled Molecule on a Metal Surface. Nature 2011, 479, 208-211.

11. Neumann, J.; Gottschalk, K. E.; Astumian, R. D. Driving and Controlling Molecular Surface Rotors with a Terahertz Electric Field. ACS Nano 2012, 6, 52425248.

12. Seldenthuis, J. S.; Prins, F.; Thijssen, J. M.; Zant, H. S. J. van der An AllElectric Single-Molecule Motor. ACS Nano 2010, 4, 6681-6686.

13. Horinek, D.; Michl, J. Surface-mounted Altitudinal Molecular Rotors in Alternating Electric Field: Single-molecule Parametric Oscillator Molecular Dynamics. PNAS 2005, 102, 14175-14180.

14. Perera, U. G. E.; Ample, F.; Kersell, H.; Zhang, Y.; Vives, G.; Echeverria, J.; Grisolia, M.; Rapenne, G.; Joachim, C.; Hla, S.-W. Controlled Clockwise and Anticlockwise Rotational Switching of a Molecular Motor. Nature Nanotechnology 2013, 8, 46-51.

15. Vives, G.; Rapenne, G. Directed Synthesis of Symmetric and Dissymmetric Molecular Motors Built Around a Ruthenium Cyclopentadienyl Tris(indazolyl)borate Complex. Tetrahedron 2008, 64, 11462-11468.

16. Vives, G.; Carella, A.; Launay, J.-P.; Rapenne, G. The Chemistry of 1,2,3,4,5pentaphenylcyclopentadienyl Hydrotris(indazolyl)borate ruthenium(II) Complexes, Building Blocks for the Construction of Potential Organometallic Molecular Motors. Coordination Chemistry Reviews 2008, 252, 1451-1459.

17. Echeverría, J.; Carreras, A.; Casanova, D.; Alemany, P.; Alvarez, S. Concurrent 
Symmetries: The Interplay Between Local and Global Molecular Symmetries.

Chemistry - A European Journal 2011, 17, 359-367.

18. Sheppard, D.; Terrell, R.; Henkelman, G. Optimization Methods for Finding Minimum Energy Paths. The Journal of Chemical Physics 2008, 128, 134106-13410610 .

19. Joachim, C. The Driving Power of the Quantum Superposition Principle for Molecule-machines. Journal of Physics: Condensed Matter 2006, 18, S1935-S1942.

20. Astumian, R. Thermodynamics and kinetics of a Brownian motor. Science 1997, 276, 917-922.

21. Joachim, C.; Ratner, M. A. Molecular Electronics: Some Views on Transport Junctions and Beyond. PNAS 2005, 102, 8801-8808.

22. Sanchez-Palencia, L. Directed Transport of Brownian Particles in a Double Symmetric Potential. Phys. Rev. E 2004, 70, 011102.

23. García-Iriepa, C.; Marazzi, M.; Zapata, F.; Valentini, A.; Sampedro, D.; Frutos, L. M. Chiral Hydrogen Bond Environment Providing Unidirectional Rotation in Photoactive Molecular Motors. J. Phys. Chem. Lett. 2013, 4, 1389-1396.

24. Ample, F.; Joachim, C. A semi-empirical study of polyacene molecules adsorbed on a $\mathrm{Cu}(110)$ surface. Surf. Sci. 2006, 600, 3243-3251.

25. Dewar, M. J. S.; Thiel, W. Ground States of Molecules. 38. The MNDO

Method. Approximations and Parameters. J. Am. Chem. Soc. 1977, 99, 4899-4907.

26. M. J. Frisch, G. W. Trucks, H. B. Schlegel, G. E. Scuseria, M. A. Robb, J. R.

Cheeseman, G. Scalmani, V. Barone, B. Mennucci, G. A. Petersson, H. Nakatsuji, M.

Caricato, X. Li, H. P. Hratchian, A. F. Izmaylov, J. Bloino, G. Zheng, J. L. Sonnenberg, M. Hada, M. Ehara, K. Toyota, R. Fukuda, J. Hasegawa, M. Ishida, T. Nakajima, Y. Honda, O. Kitao, H. Nakai, T. Vreven, J. A. Montgomery, Jr., J. E. Peralta, F. Ogliaro, M. Bearpark, J. J. Heyd, E. Brothers, K. N. Kudin, V. N. Staroverov, R. Kobayashi, J. Normand, K. Raghavachari, A. Rendell, J. C. Burant, S. S. Iyengar, J. Tomasi, M. Cossi, N. Rega, J. M. Millam, M. Klene, J. E. Knox, J. B. Cross, V. Bakken, C. Adamo, J. Jaramillo, R. Gomperts, R. E. Stratmann, O. Yazyev, A. J. Austin, R. Cammi, C.

Pomelli, J. W. Ochterski, R. L. Martin, K. Morokuma, V. G. Zakrzewski, G. A. Voth, P. Salvador, J. J. Dannenberg, S. Dapprich, A. D. Daniels, Ö. Farkas, J. B. Foresman, J. V. Ortiz, J. Cioslowski, and D. J. Fox Gaussian 09, Revision A.1; Gaussian, Inc.:

Wallingford CT, 2009. 

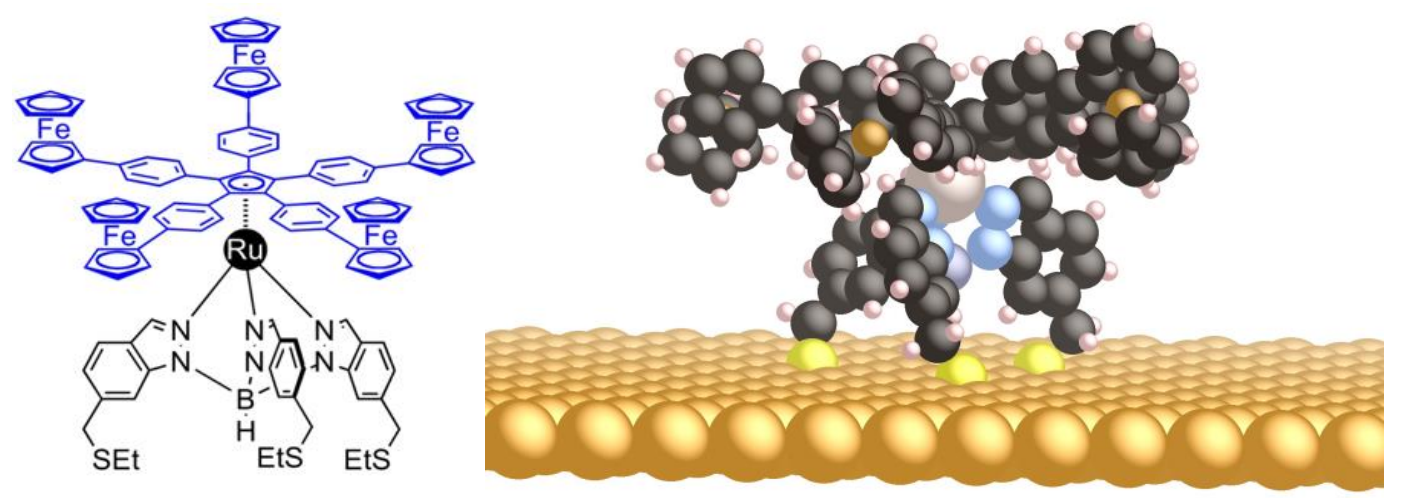

Figure 1: The detailed chemical structure (a) and the surface adsorption conformation on $\mathrm{Au}(111)(\mathrm{b})$ of the molecule-motor at the basis of this paper. 


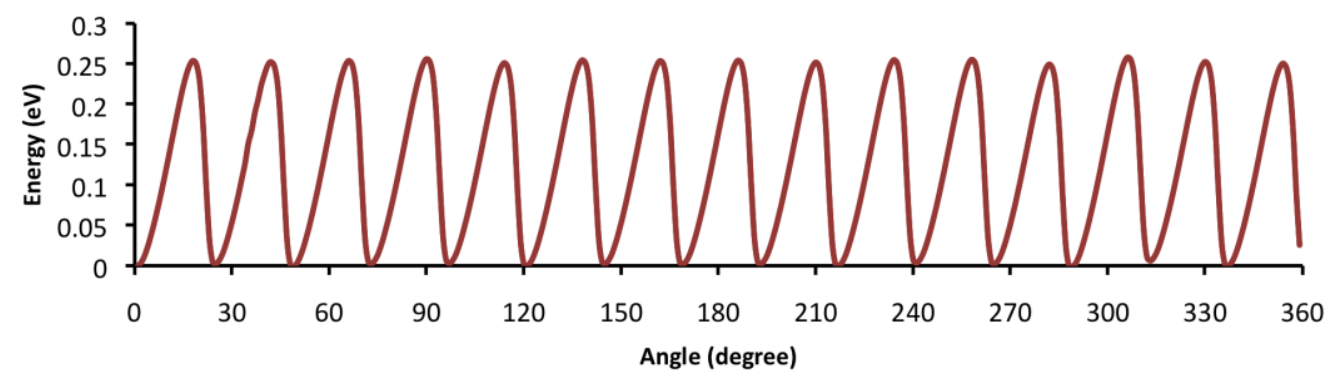

Figure 2. Asymmetric potential energy profile for a forced rotation of the molecule motor shown in Fig. 1a. Calculations were carried out with the semi-empirical ASED+ method. 

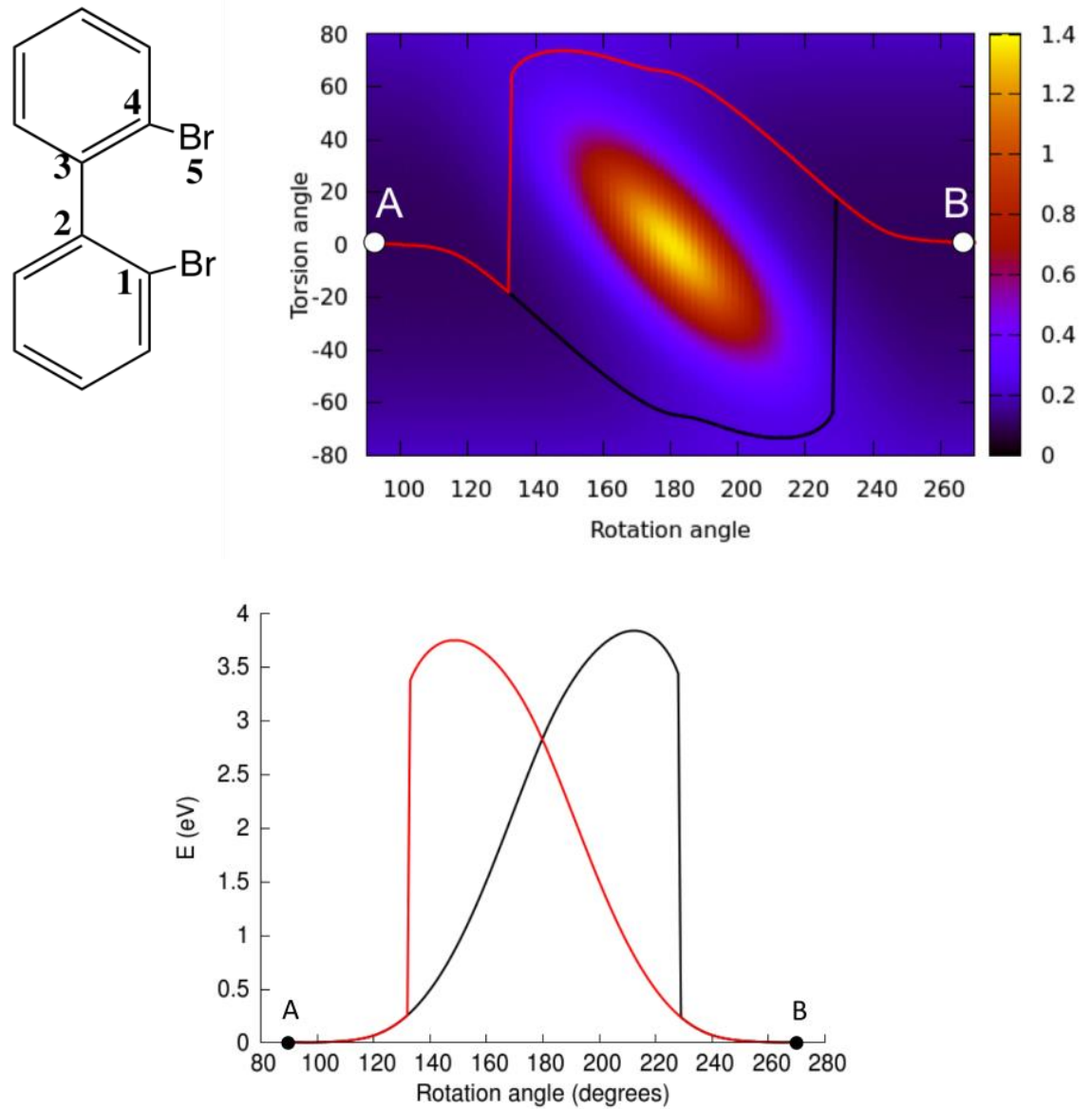

Figure 3. a) 2,2'-dibromobiphenyl with the significant atoms labelled. Rotation angle is the dihedral formed by 1-2-3-4 and torsion angle is the dihedral formed by $2-3-4-5$. b) Potential energy surface map built from the scan calculation of the torsion and rotation angles of 2,2'-dibromobiphenyl. The black line represents the value of the torsion angle for each rotation angle from $\mathrm{A}$ to $\mathrm{B}$, whereas the red line represents the value of the torsion angle for each rotation angle from B to A. The torsion angle was the only degree of freedom optimized during the calculation of both paths. The lateral colored bar is the scale for the energy in a.u. c) Potential energy profile for a forced rotation from A to B (black line) and from B to A (red line). 


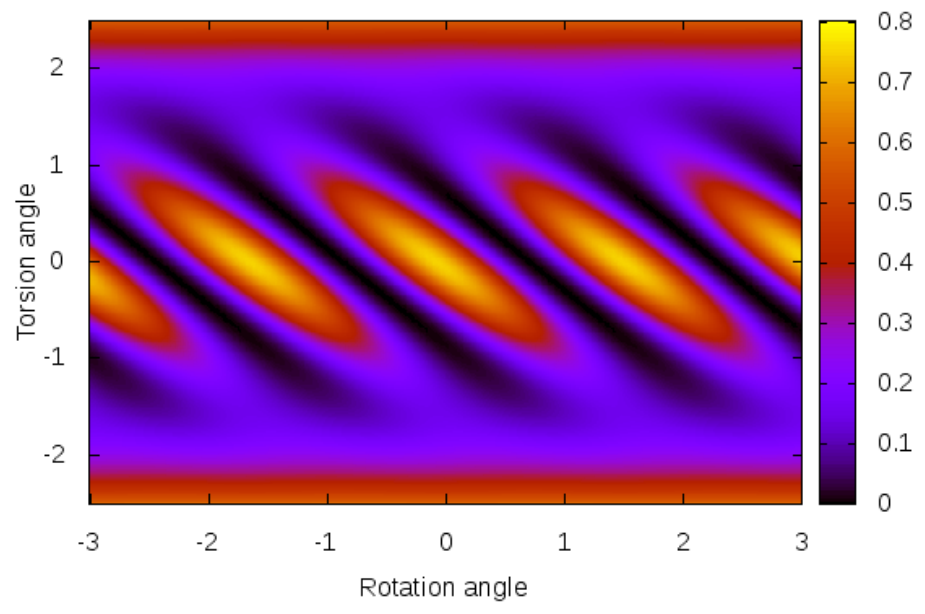

Figure 4. Potential energy surface, given by Equation 1, used in numerical simulations as the ground state of the molecule motor. The lateral bar represents the scale of $E_{0}(\theta, \varphi)$ for a value of $V_{0}=1$. 


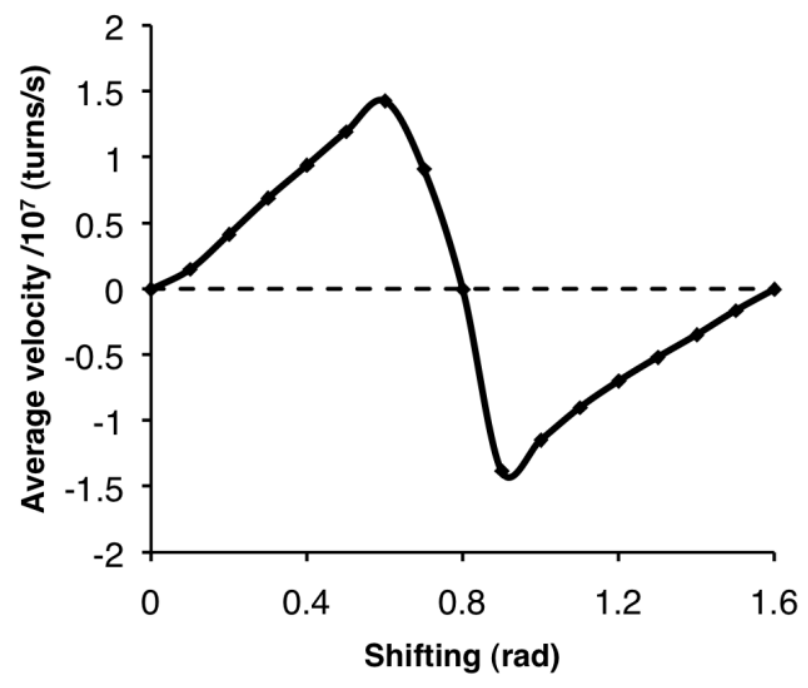

Figure 5. Effect of the electronic states shifting on the rotation efficiency and direction for a model with a symmetric ground state and a symmetric excited state. The average time between hits is $3.5 \mathrm{~ns}$ for a $10 \mathrm{~ms}$ simulation. 

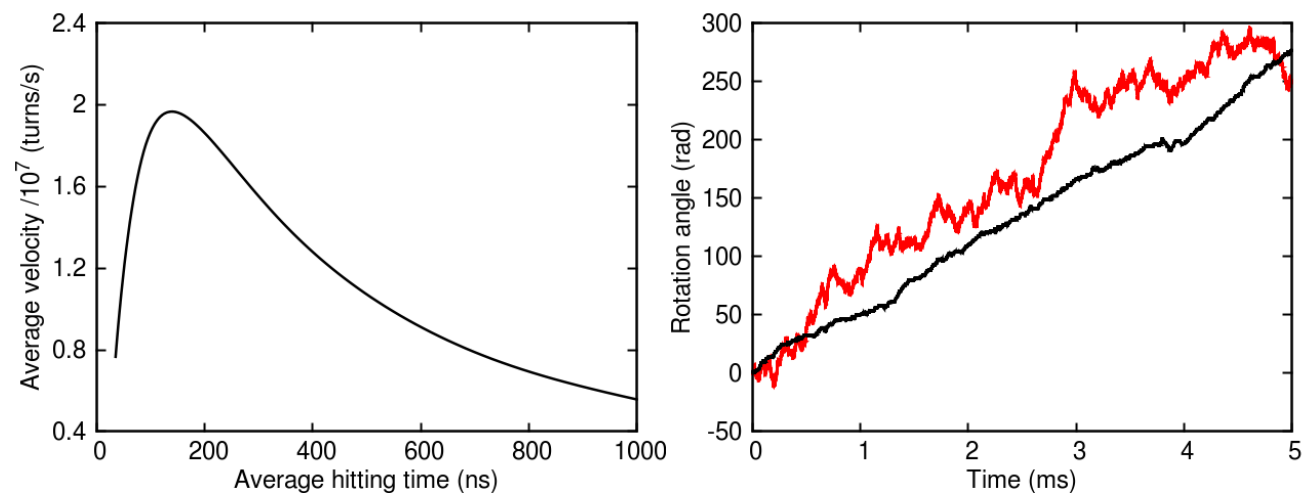

Figure 6. a) Average velocity as a function of the average time between two hits, for a model with a shifting of $0.6 \mathrm{rad}$ between electronic states. b) Angle of rotation as a function of the time. The red line is for an average hitting time of 0.35 ns whereas the black line is for an average hitting time of $7 \mathrm{~ns}$. 